\title{
Atomic Force Microscopy Studies of Human Recombinant Bone Morpho- genetic Protein OP-1
}

\author{
L. M. Siperko*, S. Chubinskaya ${ }^{\#}$, D. Rueger ${ }^{\# \#}$ and W. J. Landis*
}

*Department of Biochemistry and Molecular Pathology, Northeastern Ohio Universities College of Medicine, Rootstown, $\mathrm{OH} 44272$, "Department of Biochemistry and Section of Rheumatology, Rush Medical College, Chicago, IL 60612 and ${ }^{\# \#}$ Stryker Biotech, Hopkinton, MA 01748

Bone morphogenetic proteins (BMPs) are members of the transforming growth factor- $\beta$ (TGF- $\beta$ ) superfamily, which is composed of more than 30 homologous proteins that are biologically multifunctional. These proteins are known to induce bone and cartilage formation and have developmental roles in the induction of other mammalian tissue. Binding proteins ensure that members of the TGF- $\beta$ superfamily are folded correctly and that the TGF$\beta$ complex is targeted to the extracellular matrix (ECM) of various cells and tissues, for either storage within the tissue or activation at cell surfaces. It is thought that the ability of TGF$\beta$ to interact with cell-surface integrins, such as $\alpha_{v} \beta_{1}$, facilitates integrin signaling activation [1]. Members of the BMP family can regulate progression in osteogenic and chondrogenic lineages, supposedly through their selective affinity for specific receptor complexes [2]. Osteogenic protein 1 (OP-1), also known as BMP-7, is recognized for its ability to induce new cartilage and bone formation in vivo and its potential for the treatment of metabolic bone diseases, including osteoporosis [1]. OP-1 induces endochondral bone formation and promotes mineral formation in cultured avian growth plate chondrocytes [3]. OP-1 also plays a role in the repair and regeneration of other tissues. Thus OP-1 has diverse biological activities with implications ranging from migration, proliferation and differentiation of mesenchymal cells during embryogenesis to the repair and regeneration of tissues during postfetal life [4].

While the biological functions of this family of proteins are recognized to be diverse, BMP structure-function relationships or BMP interactions with other ECM components remains unclear. It is known, for instance, that the small extracellular binding proteins decorin and biglycan interact with TGF- $\beta$ s, inhibiting their activity [1], but mechanistic details at the molecular level have not been elucidated. This report presents preliminary results of atomic force microscopic studies that address the molecular structure of the unfolded OP-1 protein. Data obtained thus far preface investigating OP-1 interactions with small proteoglycans for the purpose of identifying specific interactive sites on the protein surface. Mature human recombinant OP-1 (Stryker Biotech, Hopkinton, MA), consisting primarily of dimers, was suspended in ethanol $(<1 \mu \mathrm{g} / \mathrm{ml})$, deposited with a syringe onto a freshly cleaved mica substrate, and allowed to dry. Topographic images were acquired under ambient laboratory conditions with a Nanoscope IIIa atomic force microscope (Digital Instruments, Santa Barbara, CA) operated in the tapping mode (TMAFM).

Figures 1 and 2 present representative TMAFM topographic images obtained of OP-1 that had been deposited on mica substrates from ethanolic suspensions of $0.1 \mu \mathrm{g} / \mathrm{ml}$ and $0.01 \mu \mathrm{g} / \mathrm{ml}$ concentrations, respectively, and allowed to dry. The major features observed in Fig. 1 are 
ordered arrays of squares that measure $\sim 50 \mathrm{~nm}$ on edge. Such a large feature indicates that agglomerates of the OP-1 form on mica from a $0.1 \mu \mathrm{g} / \mathrm{ml}$ suspension. The agglomerates are most likely aligned because they are close-packed through the influence of the highly ordered mica substrate. Ordered arrays are absent from images of $0.01 \mu \mathrm{g} / \mathrm{ml}$ suspensions of OP-1 (Fig. 2). However, isolated agglomerates can be detected across $1 \mu \mathrm{m}^{2}$ scan areas and long protein strands measuring tens of nanometers and more in length can be observed (Fig. 2, arrow). These features are caused by physical or chemical linkages (trimers and higher) of the extended protein. Many of the strands are terminated in a large globular mass, which might be composed of irregularly folded protein that occurs in suspension and then collapses to form such characteristic assemblies in the dried state.

The results shown here indicate that conformational features of OP-1 can be identified when the protein is deposited on mica and observed by TMAFM. Increasingly detailed structure can be observed at progressively lower protein concentrations. Work in progress is directed to determining precise experimental conditions necessary for obtaining higher resolution topographic images of the extended $\mathrm{OP}-1$ protein. These data will provide the basis for subsequent studies by AFM of specific sites of the protein at which putative interactions with small proteoglycans might take place. Differences in interaction sites may serve as a possible means of distinguishing between the variety of protein-ECM component and structure-function relations for TGF- $\beta$ superfamily members.

\section{References}

1. E. Piek et al., FASEB, 13 (1999) 2105

2. L. Erlacher et al., J. Bone Mineral Res., 13 (1998) 383

3. D.L. Griffith et al., Proc. Natl. Acad. Sci. USA, 93 (1996) 878

4. L.N. Wu et al., J. Cell Biochem., 67 (1997) 498

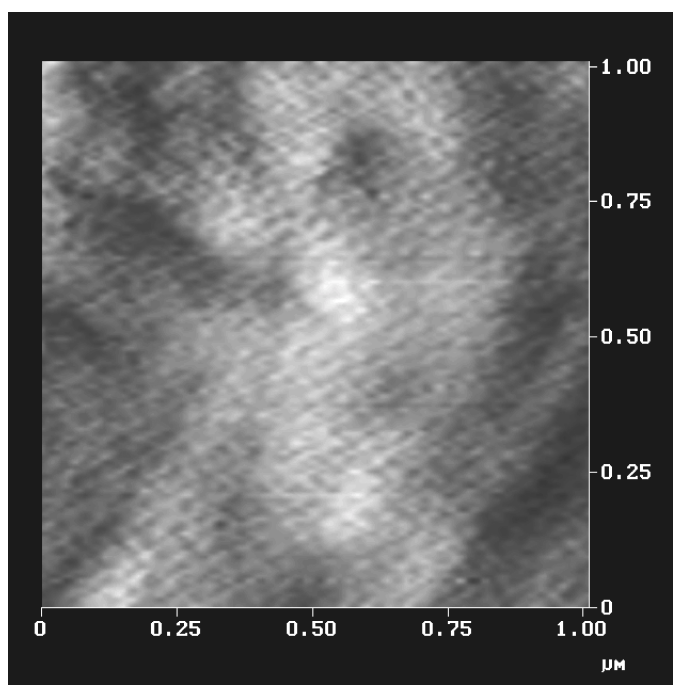

FIG. 1 - TMAFM topographic image of human recombinant OP-1 deposited on mica from a $0.1 \mu \mathrm{g} / \mathrm{ml}$ ethanolic suspension. $x, y=1.0 \mu \mathrm{m}$, $z=50 \mathrm{~nm}$.

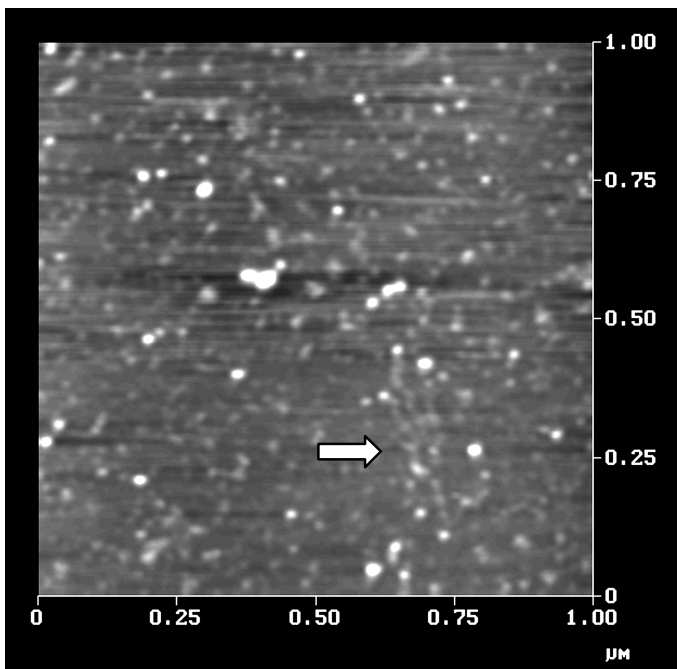

FIG. 2 - TMAFM topographic image of human recombinant OP-1 deposited on mica from a $0.01 \mu \mathrm{g} / \mathrm{ml}$ ethanolic suspension. $x, y=1.0 \mu \mathrm{m}$, $z=5 \mathrm{~nm}$. 